\title{
PRODUCTOS VEGETALES UTILIZADOS EN MADRID ENTRE LOS SIGLOS XIV Y XIX
}

\section{Luis Ramón-Laca Menéndez de Luarca}

Universidad de Alcalá de Henares

F. Javier Tardío Pato

Instituto Madrileño de Investigación y Desarrollo Rural, Agrario y Alimentario

\section{RESUMEN}

Con la intención de esbozar una historia de la cultura madrileña de las plantas a lo largo de la Edad Media y la Edad Moderna, presentamos aquí una lista de aproximadamente 300 productos vegetales citados en diversos documentos de los siglos XIV, XVI, XVII, XVIII y XIX referentes a la ciudad de Madrid, incluyendo una hipótesis de identificación de las 170 especies correspondientes a dichos productos.

PALABRAS CLAVE: productos vegetales, Madrid, Edad Media, Edad Moderna.

\section{SUMMARY}

In an attempt to describe the plant culture of Madrid of the Middle Ages and Modern Age, this paper provides a list of some 300 vegetal products cited in various documents referring to the city dated to the fourteenth, sixteenth, seventeenth, eighteenth and nineteenth centuries. The identity of the 170 plants mentioned in these documents is discussed.

KEY WORDS: vegetal products, Madrid, Middle Ages, Modern Age.

\section{INTRODUCCIÓN}

Para la realización del presente estudio, en el que tratábamos de obtener información sobre plantas citadas en Madrid durante la Edad Media y la Edad Moderna, revisamos documentos de diversa índole correspondientes a los siglos XIV, XVI, XVII, XVIII y XIX, abarcando, por tanto, un período de cerca de seiscientos años.

El documento más antiguo que localizamos en el que se mencionaran plantas en Madrid fue una ordenanza redactada en 1380 por el Consejo de la Villa 
con el fin de remediar los daños ocasionados por el ganado en los alrededores de la ciudad ${ }^{1}$. Este documento, que se refiere a los daños causados en sembrados, viñas, huertos, frutales, otros árboles, dehesas, sotos y prados, menciona cerca de tres decenas de plantas cultivadas en las huertas de Madrid. La importancia de la horticultura en el Madrid medieval queda patente en el hecho de que la multa de 30 maravedís por arrancar árboles se elevaba hasta 100 en el caso de los frutales. Cuando el ganado causaba algún daño a los árboles la multa era de 10 maravedís:

«Sy ganado mayor o menor entrare o fizyere daño en las huertas de Madrid o de su término, e lo fizyere en lo senbrado, que peche el dueño del ganado de la caloña $[\ldots]$ e sy estos ganados sobre dichos fizyeren daño en los árboles de las huertas royéndolos o quebrándolos, que peche su dueño del ganado diez maravedís por cada cabeça».

Todavía en el siglo XVI, las huertas, que se extendían alrededor de la ciudad de Madrid sobre todo en la vega del río Manzanares, ocupaban algunos solares dentro de las murallas, como puede verse en el dibujo de la ciudad realizado por Anton van den Wyngaerde hacia 1560, en el que aparece con claridad un grupo de árboles cerca de la calle de Segovia ${ }^{2}$. Generalmente, estas huertas estaban formadas por árboles de sombra, frutales, una alberca y un pozo con su correspondiente noria movida por un burro. Éste es, por ejemplo, el caso de una huerta situada entre la Casa de Campo y la ribera del Manzanares comprada por Felipe II hacia 1560; tenía esta huerta álamos blancos y negros, un pozo de noria y cincuenta árboles frutales ${ }^{3}$.

Para documentar las plantas utilizadas en Madrid en el siglo XVI, recurrimos a un documento de tipo fiscal, las alcabalas de 1592, es decir los gravámenes sobre las especias, la fruta y las hortalizas que se vendían en la ciudad ${ }^{4}$.

Para el siglo XVII, utilizamos la obra inédita de Bernardo Cienfuegos en siete volúmenes Historia de yerbas y plantas $^{5}$. De esta extensa obra, única-

1 Pérez Chozas, A., A. Millares Carlo y E. Varela Hervías (1932), Documentos del Archivo General de la Villa de Madrid, Madrid, [s.n.] p. 144-146.

2 Kagan, R. L. (1986), Ciudades del Siglo de Oro. Las vista españolas del Anton Van den Wyngaerde, Madrid, El Viso, p. 110-118.

3 Gómez Iglesias, A. (1971), «La Sagra madrileña, el Campo del Moro y la Casa de Campo», Villa de Madrid, 33, 9-20, p. 13.

4 Alvar EzQuerra, A. (1989), El nacimiento de una capital europea. Madrid entre 1561 y 1606, Madrid, Ayuntamiento de Madrid, Turner Libros, p. 244.

5 Cienfuegos, B. (1626-1631), Historia de yerbas y plantas, mss. 3.357-3.363, Biblioteca Nacional de Madrid. 
mente hemos recogido las referencias expresas a plantas vendidas o consumidas en Madrid, las cuales pueden fecharse entre 1626 y 1631.

En lo que se refiere al siglo XVIII, consultamos la Flora española de José Quer y Casimiro Gómez-Ortega, obra en la que se mencionan una gran variedad de árboles y arbustos cultivados durante el siglo XVIII en el «circuito de Madrid», en «jardines de curiosos» o en la «Real Casa del Campo» ${ }^{6}$. Al tratarse de una obra botánica, son más bien anecdóticas las referencias a plantas cultivadas o útiles, aunque algunas de ellas tienen un indudable interés, como por ejemplo la alusión al cultivo del alfónsigo (Pistacia vera L.) en Madrid y sus alrededores.

En cuanto al siglo XIX, las fuentes empleadas fueron dos, por una parte, los inventarios inéditos redactados por Mariano Lagasca y sus discípulos en las herborizaciones llevadas a cabo alrededor de la ciudad entre 1802 y 1818 (signs. I, 12, 4, 2 y I, 27, 8, 4-14, Archivo del Real Jardín Botánico) y, por otra, dos listas de los productos despachados en las cinco puertas de entrada de Madrid en 1847 y $1848^{7}$. En los inventarios elaborados por Lagasca y sus discípulos se reseñan, aparte de las especies silvestres, las plantas cultivadas en las huertas madrileñas. A mediados del siglo XIX existían aún fuera de la cerca de la ciudad de Madrid numerosas huertas: 79 huertas particulares, con una superficie de 506 fanegas, y 8 pertenecientes al Patrimonio Real, con una superficie de 444 fanegas; un total aproximado de 325 hectáreas $^{8}$. En cuanto a los productos gravados por aranceles y portazgos en las puertas de Madrid, hemos podido entresacar de las listas citadas más de dos centenas de productos de origen vegetal.

En el Apéndice 1 se presenta una lista de especies con sus correspondientes nombres vulgares, que son los que aparecen normalmente en la documentación estudiada, excepto en la Flora de Quer y los inventarios de Lagasca, trabajos de índole botánica que recogen en primer lugar el nombre científico de la especie. Para la elaboración de las hipótesis, en cada caso particular, nos hemos servido de diversas obras sobre nombres populares y usos de las plantas ${ }^{9}$. Para evitar la reiteración y facilitar la comprensión al lector, en el apén-

6 Quer, J. y C. Gómez Ortega (1762-1784), Flora española, Madrid, Joachin Ibarra.

7 Madoz, P. (1847), Diccionario geográfico-estadístico-histórico de España y sus posesiones de ultramar, vol. 10, Madrid, [s.n.], p. 1037-1059 y 1065-1072.

8 MAdOZ (1847), p. 985. Por Real Orden del 9 de diciembre de 1852 publicada en la Gaceta de Madrid el 28 del mismo mes y año, una fanega de Madrid equivalía a 3.423,8121 metros cuadrados.

9 Chich-Pulido, M. y C. FernÁndez-López (1993), Nombres castellanos de plantas vasculares en el Colmeiro (1885-1889), Jaén, Facultad de Ciencias Experimentales. FONT Quer, P. (1990), Plantas medicinales. El Dioscórides renovado, Barcelona, Editorial Labor. 
dice, se han sustituido las citas originales de los documentos mencionados por los siguientes códigos: XIV ${ }^{10}, \mathrm{XVI}^{11}, \mathrm{XVII}^{12}, \mathrm{XVIII}^{13}, \mathrm{XIX}^{14}, \mathrm{XIXbis}^{15}$. Asimismo, cuando ha sido posible, hemos incluido el uso más probable dado a cada especie y la forma de obtención de las mismas, mediante cultivo, recolección silvestre o importación. Para ello hemos utilizado los siguientes códigos: $\mathrm{AA}=$ alimentación animal; $\mathrm{AH}=$ alimentación humana; $\mathrm{I}=$ industriales $\mathrm{y}$ artesanales, incluyendo perfumeras, cosméticas, textiles, tintóreas y curtientes y leñas y combustibles; $\mathrm{M}=$ medicinales, veterinarias y tóxicas; $\mathrm{O}=$ ornamental; cult. $=$ cultivada, silv. $=$ silvestre, imp.$=$ importada .

\section{RESULTADOS Y DISCUSIÓN}

Con los nombres citados en la documentación utilizada, obtuvimos un total de 170 especies vegetales, aunque el número de productos superó los 300 , ya que de muchas especies se citaban varios productos. Como cabía esperar, se observó un aumento progresivo del número de productos a lo largo del tiempo, con cerca de 30 en el siglo XIV y de 80 en el XVI y más de 200 en el XIX.

Si examinamos las plantas citadas en el siglo XIV, podemos decir que, en esta época avanzada de la Edad Media, las huertas madrileñas se caracterizaban por un notable arcaísmo, ya que no se mencionan plantas típicas de la cultura andalusí, como las alcachofas, las berenjenas o las espinacas. En efecto, entre las hortalizas se cultivaban arvejas - guisantes-, garbanzos, habas, coles, nabos, melones, pepinos, cohombros, lechugas, puerros y zanahorias, mientras que entre los frutales, albaricoqueros, almendros, cerezos, duraznos, granados, higueras, membrillos, nogales, olivos, manzanos, perales - se menciona una variedad cermeña - y vides. Llama la atención el cultivo del agraz, hoy casi perdido, del azafrán, que en la actualidad se ha desplazado a la Mancha, y del cohombro, un tipo de melón de forma alargada y retorcida pero de sabor semejante al pepino, hoy desaparecido. Sorprende la ausencia del ajo y la cebolla, pero es evidente que el hecho de que estas plantas no se citen en

MabBerley, D. J. (2000), The plant-book, Cambridge, Cambridge University Press. MorALES, R. et al. (1996), Nombres vulgares II, Madrid, Real Jardín Botánico, CSIC.

10 PÉrez ChOZAS, A. et al. (1932).

11 Alvar EZQuerra, A. (1989).

12 Cienfuegos, B. (1626-1631).

13 Quer, J. y C. Gómez OrTEGA (1762-1784).

14 Mss. I, 12, 4, 2 y I, 27, 8, 4-14, archivo del Real Jardín Botánico, CSIC, Madrid.

15 Madoz, P. (1847). 
el documento empleado no prueba que no fueran cultivadas en Madrid en aquella época.

Dos siglos más tarde, la lista de hortalizas se ha completado y enriquecido notablemente con referencias a espinacas, acelgas, rábanos, repollos, berzas, alcachofas, cardo, arvejones, ajos, cebollas y espárragos. Además, hay novedades procedentes de las colonias, como los pimientos, la coca de Levante, que se empleaba como planta ictiotóxica, para pescar, o el palo de las Indias, que seguramente se utilizaba para combatir la sífilis. Merece destacarse, además, la referencia a la dragontea, cuyo uso nos revelará algunos años más tarde Cienfuegos. Desde el punto de vista filológico, resulta notable la expresión utilizada para referirse al alazor, zafranromín almocaser, arabismo que, según Federico Corriente (com. pers.), significa «azafrán cristiano rizado». El alazor se vendía probablemente como sucedáneo del verdadero azafrán (Crocus sativus L.), como señala ya Andrés de Laguna en sus comentarios a la Materia medica de Dioscórides, quizá para teñir, e incluso como colorete o arrebol, como indica la Flora de Quer ${ }^{16}$. Volviendo al siglo XVI, se citan legumbres no mencionadas en el siglo XIV, como las lentejas, y cereales como el arroz. Llama la atención la variedad de plantas útiles para ensaladas vendidas en Madrid, como achicorias, berros, cardillos, escarolas y lechugas - se mencionan expresamente «otras yerbas para ensaladas»-, las tres primeras procedentes de recolección silvestre. Igualmente, se citan cinco especies de cítricos, cidras, limas, limones, naranjas y toronjas. Lo mismo cabe decir de otras frutas, como azufaifas, bellotas, guindas, madroños, manzanas camuesas, moras de moral y nísperos. De otras frutas reseñadas ya en el siglo XIV se mencionan nuevas variedades, como por ejemplo albérchigos, duraznos y melocotones, que se refieren seguramente a diferentes variedades de Prunus persica (L.) Batsch. Además, en esta época en Madrid se vendían otras plantas destinadas a la alimentación humana, como regaliz, altramuces y chufas, y animal, como algarrobas, alpiste y cañamones, y asimismo una gran variedad de condimentos, como azúcar, canela, clavo, comino rústico, cilantro, jengibre, hierbabuena, pimienta, nuez moscada y perejil. Se citan también criadillas o turmas de tierra y frutos secos, como orejones, probablemente de albaricoques y melocotones, pasas, avellanas, castañas y piñones, y dulces de frutas, como calabazate, carne de membrillo y diacitrón, a los que en el siglo XIX se añadirán mostillo, mermeladas y letuario, una especie de mermelada. Con todos estos datos, puede decirse que el siglo XVI fue, en Madrid, una

16 Font Quer, P. (1990), p. 855-856. 
época más favorable en lo que se refiere a la alimentación y al uso de las plantas, ya fueran éstas cultivadas o no en la propia ciudad.

El siglo XVII fue, en cambio, una época de dificultades y escasez de alimentos. Así, Bernardo Cienfuegos menciona la especulación con el arroz en Madrid en 1631:

«En España cuidan poco desta semilla y este año 1631 lo avían ocultado de tal manera los mercaderes y tratantes que la traen de Valencia a Madrid que no se hallaba a dos reales la libra. Vino la desgracia (si no fue castigo de Dios) y abrasando por un incendio fortuito sus casas, haziendas, mercadurías y personas a $7 \mathrm{de}$ julio de 1631 en la quarta parte de la plaza de Madrid que arruynó el incendio sin lo mucho que se abrasó se sacaron del centro de la tierra más de cien talegas de arroz de a dos fanegas; pero luego bolvieron a desaparezer como humo aunque escapadas del fuego» ${ }^{17}$.

Las malas cosechas y la consiguiente carestía de alimentos básicos como el pan llevan a Cienfuegos a sopesar la posibilidad de utilizar plantas silvestres no consumidas tradicionalmente, como los abrojos:

«En Madrid, después de el [trigo] de leche y candial, entra el de Guadalajara y Vallecas, Pinto, Móstoles y su comarca, el de Toledo y su trechel, luego por penúltimo el de Guadarrama, y por peor el que amassan los panaderos de dentro Madrid rebolviéndolo con lo remolido de los panezillos candeales» ${ }^{18}$.

«Al tiempo que escrivo esto que es el mes de noviembre de año de Nuestro Salvador 1630 es tanta la necesidad de pan y tan común la carestía y falta dél que muy malo, negro y rebuelto de malas semillas se vende en Madrid, corte del Rey Philippo $4^{\circ}$, a trece y catorce quartos cada pan de dos libras, y vale una fanega de harina de las calidades dichas sesenta y seis reales y no se halla por el dinero. En semejantes años como éste podía estimarse el fruto destos tribulos o abrojos para remediar la falta» ${ }^{19}$.

La falta de trigo llevó consigo la subida del precio del almidón, del que una libra [de almidón de trigo candeal] valía en 1630 dos reales ${ }^{20}$. En su lugar se utilizaban raíces de dragontea, empleándose el residuo sólido sobrante para cebar lechones:

\footnotetext{
17 Cienfuegos, B., ms. 3357: xvii -xviii.

18 Cienfuegos, B., ms. 3357: 81-82.

19 Cienfuegos, B., ms. 3363: 626-627.

20

Cienfuegos, B., ms. 3357: 357.
} 
«El Rey don Felipe II prohibió el usso del almidón y las mugeres lo hazían mucho mejor, más tiesso, más blanco y transparente de rayzes de tragontía o serpentaria mayor sacando su leche por expressión y dexándola secar. El buruxo que quedaba apetecían muchíssimo los animales y se zevaban con él los lechones y engordaban» ${ }^{21}$.

Asimismo, gracias al testimonio de Cienfuegos, sabemos que, durante el siglo XVII, iban llegando a Madrid plantas procedentes de las colonias. Por ejemplo, el autor de la Historia de yerbas y plantas menciona diversas especias en manos de «caballeros indianos» en 1627, que se usaban para poner con el chocolate, como por ejemplo «la canela de la provincia de Yucatán» conocida por los madrileños como canela de Icabo:

«En los despojos de un cavallero indiano de los que llaman criollos, que son hijos de españoles y indias, que murió en Madrid, vinieron a mis manos diversos frutos, raízes i gomas traídas de la India Occidental, como fueron raíces de contrayerva, cacao, achote i su semilla, diversas semillas de pimientos, pepitas de quina, goma del mismo árbol, otro fruto que se llama esponja o esponjilla, y otro que paresze tortuga i concha de ella o de erizo marino, con tuétano o meollo dentro de los quales Dios queriendo escribiremos. Havía una corteza que holía a canela i tenía su mesmo sabor i gusto con aromaticidad i picante más suave que el de la pimienta, de color ceniza o blanquezino, gruessa de un real de a quatro algo encorvada o arrugada (como unas tejuelas que las monjas haszen de pasta de alcorça). Destas corteszas avía quantidad de media libra, i todo estaba sin título o intitulata alguna. El gusto, olor i forma, como e dicho, es de canela blanquezina o cinericia, aunque gruessa i no tan arrollada. A los principios me pareció la corteza que describe Monardes en el capítulo 26 del libro I con nombre de leño aromático, pero hallándole olor, sabor i aromaticidad de canela, y rebolviendo los papeles hallé que decía 'cosas buenas para poner en el chocolate'. Acudí a un amigo que tenía en esta Corte que aunque sacerdote (quod indignum) trataba en cosas de la India como eran pita zarza, cacao, añil grana y otras cosas como chocolate en cajas y tortillas para vender y beber fresco con grandes ventajas y gusto de los chupadores deste licor y sorbedores de tabaco, i mostrándole esta corteza de la qual aún tengo un pedazo en mi posada, luego como la vio me dixo: esto es canela de Icabo, buena para poner con las demás especies aromáticas como son clavos, gengibre, macias nuezes y otras» ${ }^{22}$.

En cuanto a las patatas, a las que se refiere con los nombres de Aradichna de Teofrasto o papas del Perú, Cienfuegos asegura haberlas visto por primera vez en la cartuja del Paular (Rascafría, Madrid):

21 Cienfuegos, B., ms. 3357: 358.

22 Cienfuegos, B., ms. 3357: 865-866. 
«Con el nombre que esta planta se conoze en España es con el de turmas de la India. Donde primero la vi fue en la huerta de los cartujos del Paular en Segovia» ${ }^{23}$.

Según Cienfuegos, las patatas se vendían en Madrid con el nombre de papas y eran utilizadas por «los indianos y los mismos naturales [los madrileños]» para guisados ${ }^{24}$. Cienfuegos menciona asimismo el consumo de las criadillas de tierra, las cuales corresponderían, en nuestra opinión, a la especie Terfezia arenaria (Moris) Trappe, a pesar de que Pío Font Quer ${ }^{25}$ opinaba que Cienfuegos se refería a las trufas - Tuber brumale Vittadini-

«Aunque menores [que las de Murcia y Extremadura] las [turmas de tierra] de la tierra de Madrid son muy gustosas» ${ }^{26}$.

Según Cienfuegos, se comían en esta época en España diversas setas, como los lobitos - Boletus sp.- igualmente mencionados por Laguna en su versión de la obra de Dioscórides ${ }^{27}$, los hongos mizclos o almizcleños - Lactarius deliciosus (L.: Fr.) Gray_- las orejas, cagarrias o crespillas - Morchella esculenta (L.) Pers. - y los hongos de olmos - Agrocybe aegerita (Brig.) Singer o quizá Pleurotus ostreatus (Jacquin: Fr.) Kummer ${ }^{28}$. En Madrid se recolectaban las setas de cardo — Pleurotus eryngii (DC.: Fr.) Quél.—y las de pie azul —Lepista nuda (Bull.: Fr.) Cooke o L. personata (Bull.: Fr.) Cooke-:

«Esta diferencia [de pie azul] comen la gente del campo con mucha seguridad, y aún se venden públicamente en Madrid. El año 1626, por el septiembre, hubo muchas aguas y después muchos soles, con que salieron muchas setas en los prados y sotos de Manzanares. Costó a muchos la vida y familias enteras murieron sin remedio, pero no an escarmentado, que cada día las comen sin rezelo alguno, sin hazer distinción de las de cardo o prado» ${ }^{29}$.

Ya Laguna había mencionado el hecho de que en Madrid se vendían los $\operatorname{lobitos}^{30}$. Además, según Cienfuegos, se comían en Madrid una seta que se

23 Cienfuegos, B., ms. 3359: 404r.

24 Cienfuegos, B., ms. 3357: 499.

25 FONT QueR, P. (1990), p. 23.

26 Cienfuegos, B., ms. 3359: 409v-410r.

27 FONT QUeR, P. (1990), p. 16.

28 Cienfuegos, B., ms. 3359: 433-434v.

29 Cienfuegos, B., ms. 3359: 437r.

30 FONT QUER, P. (1990), p. 16. 
criaba «en los trigos, de hechura esquinada», que no hemos podido identificar, y otra que nacía «al pie de las cañahierbas o férulas», que se refiere, sin duda, a Pleurotus eryngii (DC.: Fr.) Quél. var. ferulae Lanzi ${ }^{31}$. Cienfuegos menciona algunas plantas medicinales, que vendían unas «mujercillas» junto a la iglesia de Santa Cruz, y que se traían a veces de otras ciudades, como ocurría por ejemplo en el caso del ombligo de Venus procedente de Segovia:

«Muchas vezes, en Madrid, en aquellas mugercillas que venden yervas junto a Santa Cruz, e hallado vendiendo por yerva mora la flámula, dulciamara o sigillo de Santa María, aunque más me quadra el nombre de dulcamara flámula, o especie de vid, y echándoseles a rodar me dizen los boticarios la compran por yerva mora. Miren qué efecto harán los refrigerantes con ella» ${ }^{32}$.

«En Madrid no la e visto [el ombligo de Venus] si no es en un tejado y quatro o cinco hojas en la pared de la iglesia de La Merced, a donde es fuerza traherla de Segovia que se halla en abundancia» ${ }^{33}$.

Vale la pena reseñar el uso del eleboro en Madrid para envenenar las flechas de caza citado por Cienfuegos. Sin duda, se trata del Veratrum album L., planta que recibe igualmente el nombre de hierba de ballesteros ${ }^{34}$ :

«Su Magestad tiene un hombre que le sirve en la caza, el qual oy día vive en Fuencarral, aldea de Madrid, el qual haze la confección del eleboro para las flechas con gran eminencia ${ }^{35}$.

Otros ejemplos de plantas que permiten imaginar el ambiente de Madrid en aquella época son, por ejemplo, las «hojas de la Consiglio de Roelio en que comunmente [venían] a Madrid embueltas las pellas de manteca de vacas ${ }^{36}$, aunque no podamos decir a qué planta se refería Cienfuegos, y, finalmente, como ejemplo de planta ornamental, el Piso o disante cordato, halicacabo o besicos de monja de Gregorio de los Ríos, seguramente Cardiospermum halicacabum L.:

«En Madrid la crían en los jardines sólo por hermosura y verdura que trepe y enrrame las paredes y cenadores. En la calle Imperial de Madrid un barbero la a

\footnotetext{
31 Cienfuegos, B., ms. 3359, f. 437r-437v.

2 Cienfuegos, B., ms. 3357: 1155.

Cienfuegos, B., ms. 3357: 1175, 1178.

34 FONT Quer, P. (1990), p. 881.

35 Cienfuegos, B., ms. 3359, f. 382r.

36 Cienfuegos, B., ms. 3357: 1086.
} 
criado en un artesón muchos años enrredando con ella una ventana. Ninguno que yo sepa ussa della para medicina ${ }^{37}$.

En cuanto al siglo XVIII, puede destacarse el cultivo del acerolo en las huertas de la Casa de Campo y del alfónsigo en varios lugares de la ciudad:

«Se halla ya connaturalizado [el alfónsigo o pistacho] en algunas partes de España, como en el Real Jardín Botánico, en el que posee el Excelentísimo Señor Duque del Infantado en el lugar de Chamartín, cerca de esta corte, y en varias huertas de curiosos. En la villa de Getafe, a dos leguas de Madrid, se ven dos árboles de esta especie muy reviejos, que se conservan de tiempo inmemorial al abrigo de una pared que cae al mediodía en el patio de una casa, que llaman por eso la casa de los alfónsigos en la calle mayor» ${ }^{38}$.

En el siglo XIX, llama la atención la presencia en las huertas madrileñas del armuelle, de las borrajas, de la chirivía, del mastuerzo y de la verdolaga, seguramente reliquias de antiguos cultivos. Sorprende, asimismo, la venta de acerolas, fruta que puede considerarse hoy en Madrid una rareza, e igualmente de érrax - huesos de aceitunas-, combustible empleado tradicionalmente en Madrid para los braseros:

«Mandó [doña Lucrecia] que aderezaran una sala que caía al jardín, adornándola de turquesadas alfombras, almohadas y sillas bordadas, ricas y costosas láminas, varias pinturas, lustrosos y grandes escritorios, dos braseros de plata, colmados de menudo y bien encendido erraj ...» ${ }^{39}$.

Lo mismo cabe decir del alcacer, o cebada verde para pasto ${ }^{40}$ y del sen, utilizado tradicionalmente como laxante, cuyo cultivo fue introducido a finales del siglo XVIII por Antonio Palau, catedrático del Real Jardín Botánico ${ }^{41}$. Aparte de estos rasgos arcaicos, una época tan cercana a la actualidad como es el siglo XIX significa, como es lógico, la llegada a Madrid de una gran diversidad de productos vegetales. Así, entre otros, aparecen hortalizas, como

37 Cienfuegos, B., ms. 3363: f. 263.

38 Quer, J. y C. GÓMEZ OrTEGA (1762-1784), vol. 6, p. 364.

39 Carvajal y SAAVEDRA, M. de (1993 [1663]), Navidades de Madrid y noches entretenidas, en ocho novelas, edición, prólogo y notas de C. Soriano, Madrid, Comunidad de Madrid, p. 18.

40 CORRIENTE, F. (1999), Diccionario de arabismos y voces afines en iberromance, Madrid, Gredos, p. 128a.

41 Font Quer, P. (1990), p. 353. 
apio, batatas (de Málaga), berenjenas, grelos, judías (probablemente de la Granja o del Barco), rábanos o tomates; condimentos como alcaparras, alcaravea, alholva, anís, comino, hinojo, laurel, orégano, perejil o tomillo; aceitunas de diferentes clases y un sinfín de plantas diversas, como algarrobas, cacahuetes, dátiles, grosellas, palmitos e higos chumbos; uvas y vinos de diversas clases; infusiones como café, té o valeriana; harinas de algarrobas, de arroz, de maíz y de sagú; forrajes como alfalfa, avena, cebada, almortas, mijo o yeros; plantas curtientes o tintóreas como añil, rubia o zumaque; finalmente, otros productos de uso industrial como árnica, acebo, aceite de coco y de enebro o miera.

\section{CONCLUSIÓN}

Entre los siglos XIV y XIX se produjo en Madrid un enriquecimiento progresivo del número de productos vegetales utilizados, que se corresponde con la lógica evolución histórica, la mejora de los caminos y los medios de transporte y una mayor estabilidad de las condiciones de vida de la capital de España a partir de finales de la Edad Media. A pesar de que hacia 1600 aparecen en Madrid algunas novedades procedentes de las colonias, la ciudad continúa evidenciando su carácter tradicional, con la aparición de algunos elementos arcaicos. Este carácter se mantiene todavía en el siglo XIX, cuando se cultivaba aún en la capital de España una gran diversidad de hortalizas. Sin embargo, el catálogo de productos vegetales es ya en esta época fiel reflejo de las crecientes necesidades de la sociedad contemporánea ${ }^{42}$.

42 Presentamos en este trabajo parte de los datos históricos reunidos durante el desarrollo del proyecto de investigación «Etnobotánica alimentaria en la Comunidad de Madrid» (proyecto $07 \mathrm{M} / 0027 / 00$, financiado por la Dirección General de Investigación de la Comunidad de Madrid), llevado a cabo entre 2000 y 2002 por un equipo de investigadores del Instituto Madrileño de Investigación Agraria y Alimentaria (Comunidad de Madrid) y del Real Jardín Botánico de Madrid (CSIC). Durante el transcurso del proyecto, comparamos la información obtenida directamente de los informantes con diversas fuentes históricas, con el fin de establecer una posible relación entre los productos tradicionales consumidos en la actualidad y los disponibles en los mercados madrileños en el pasado. Agradecemos la ayuda prestada durante la elaboración de este trabajo por Ramón Morales, Manuel Pardo de Santayana y Pilar San Pío, investigadores del Real Jardín Botánico (CSIC), de Federico Corriente, profesor de la Universidad de Zaragoza, y de Higinio Pascual, investigador del Instituto Madrileño de Investigación Agraria y Alimentaria. 


\section{Apéndice 1}

\section{Lista de especies y sus correspondientes nombres de productos vegetales ${ }^{43}$}

Adiantum capillus-veneris L.: culantrillo (XIXbis). M silv.

Agave americana L.: pita zarza (XVII); cardón (XIXbis). I cult.

Agrocybe aegerita (Brig.) Singer o Pleurotus ostreatus (Jacquin: Fr.) Kummer: hongo de olmos (XVII). AH silv.

Allium cepa L.: cebolla (XVI, XIXbis). AH cult.

A. porrum L.: puerro (XIV, XVI). AH cult.

A. sativum L.: ajo (XVI, XIXbis); ajo seco (XIXbis). AH cult.

Anamirta cocculus (L.) Wight \& Arn.: coca de Levante para matar peces (XVI). MD imp.

Ananas comosus (L.) Merr.: fruta en piña de América (XIXbis). AH imp.

Annona cherimola Miller: chirimoya (XIXbis). AH cult.

Apium graveolens L.: - (XIX). AH cult.

Arachis hypogaea L.: cacahué (XIXbis); cacahuet (XIXbis). AH cult.

Arbutus unedo L.: madroño (XVI, XVIII, XIXbis). AH silv.

Arnica montana L.: árnica (XIXbis). M silv.

Artemisia abrotanum L.: - (XIX). M. cult.

Asparagus acutifolius L.: -(XIX); espárrago de Trujillo (XIXbis). AH silv.

A. officinalis L.: espárrago (XVI); - (XIX); espárragos de Calera, de jardín, de la Mancha y de Seseña (XIXbis). AH cult. Atriplex hortensis L.:-(XIX). AH cult. Avena sativa L.: avena (XIXbis). AH cult.
Beta vulgaris L.: - (XIX); acelga (XVI). AH cult.

Bixa orellana L.: achote (XVII). I imp.

Boletus sp.: lobitos (XVII); setas estrangeras (XIXbis). AH silv.

Borago officinalis L.: - (XIX). AH cult.

Brassica juncea (L.) Czern.: - (XIX). AH cult.

B. napus L.: - (XIX); nabo (XIV, XVI, XIXbis). AH cult.

B. nigra (L.) W. D. J. Koch: - (XIX). AH cult.

B. oleracea L.: col (XIV); berza (XVI); repollo (XVI, XIXbis); - (XIX); coliflor (XIXbis). AA y AH cult..

B. rapa L.: rábano (XVI). AH cult.

B. rapa L. subsp. campestris (L.) A. R. Clapham: - (XIX). AH cult.

Caesalpinia paraguariensis (Parodi) Burkart: palo de las Indias (XVI). M imp.

Camellia sinensis (L.) Kuntze: té (XIXbis). AH imp.

Canella winterana (L.) Gaertner: canela de Icabo (XVII). AH imp.

Cannabis sativa L.: cañamón (XVI, XIXbis); - (XIX). AA cult.

Capparis spinosa L.: alcaparra (XIXbis). AH cult.

Capsicum annuum L.: pimiento de Indias (XVI); pimiento (XVII); - (XIX); pimiento de la Rioja (XIXbis); pimiento molido (XIXbis). AH cult.

Cardiospermum halicacabum L.: besicos de monja (XVII). O cult.

43 Para la nomenclatura, utilizamos el criterio de Mabberley, D. J. (2000). 
Carthamus tinctorius L.: zafranromín almocaser (XVI); alazor (XIXbis); flor de alazor (XIXbis). I cult.

Carum carvi L.: - (XIX); alcarabea (XIXbis). AH cult.

Cassia obovata Collad.: hoja de sen (XIXbis). M cult.

Castanea sativa L.: castaña (XVI); castaña mondada y apilada (XIXbis); castaña verde (XIXbis). AH cult.

Ceratonia siliqua L.: garrofa (XIXbis). AA cult.

Chamaerops humilis L.: palmito (XIXbis). AH silv.

Cicer arietinum L.: garvanço (XIV); garbanzo (XVI, XIXbis); garbanzo tostado (XIXbis). AH cult.

Cichorium endivia L.: escarola (XVI). AH cult.

C. intybus L.: achicoria (XVI); chicoria amarga (XIX). AH cult.

Cinchona calisaya Wedd.: calisaya (XIXbis). AH y M imp.

Cinnamomum verum J. Presl: canela (XVI); canela de Ceilán, de Holanda, de la China y de Manila (XIXbis). AH imp.

Citrus aurantifolia (Christm.) Swingle: lima (XVI, XIXbis); limas de Murcia y de la Vera (XIXbis). AH cult.

C. $\times$ aurantium L.: naranja (XVI, XIXbis); naranjas de Murcia y de la Vera (XIXbis); hoja de naranjo (XIXbis). AH e I cult.

C. $\times$ limon (L.) Osb.: limón (XVI, XIXbis); limones de Murcia y de la Vera (XIXbis). AH cult.

C. maxima (Burm.) Merrill: toronja (XVI). AH cult.

C. medica L.: cidra (XVI, XIXbis); diacitrón (XVI). AH cult.

Cocos nucifera L.: aceite de coco (XIXbis). M imp.
Coffea arabica L.: café (XIXbis). AH imp.

Coriandrum sativum L.: culantro (XVI); - (XIX); cilantro (XIXbis). AH cult.

Corylus avellana L.: avellana (XVI); avellano (XVIII); avellana en casco (XIXbis); avellana tostada y mondada (XIXbis). AH cult.

Crataegus azarolus L.: azerola (XVIII); acerola (XIXbis). AH cult.

Crocus sativus L.: açafrán (XIV); azafrán (XVI, XIXbis). AH cult.

Cucumis melo L.: melón (XIV, XVI); (XIX); melones de Añover, de la tierra de Madrid, de Madrid y de Valencia (XIXbis). AH cult.

C. melo L. subsp. flexuosus (L.) Grab.: cogombro (XIV); cohombro (XVI). AH cult.

C. sativus L.: pepino (XIV, XVI, $\mathrm{XIXbis);} \mathrm{pepino} \mathrm{estrangero} \mathrm{en} \mathrm{vinagre}$ (XIXbis). AH cult.

Cucurbita maxima Duchesne ex Lam.: calabazate (XVI); calabaza (XIXbis). AH cult.

C. pepo L.: - (XIX). AH cult.

Cuminum cyminum L.: comino (XIXbis). AH cult.

Cydonia oblonga Miller: membrillo (XIV, XVI, XIXbis); carne de membrillo (XVI); - (XIX). AH cult.

Cynara cardunculus L.: cardo (XVI); hierba para cuajar (XVI); flor de cardo (XIXbis). AH cult.

C. scolymus L.: alcachofa (XVI, XIXbis). AH cult.

Cyperus esculentus L.: chufa (XVI, XIXbis). AH

Daucus carota L.: açanoria (XIV); zanahoria (XVI); - (XIX). AH cult.

Dracunculus vulgaris Schott: taragontía (XVI); tragontía o serpentaria mayor (XVII). AA e I cult. 
Ficus carica L.: figo (XIV); higo (XVI); - (XIX); breva (XIXbis); higo estrangero (XIXbis); higos pasos de la tierra, de Málaga, de Murcia y de Valencia (XIXbis); higo verde (XIXbis). AH cult.

Foeniculum vulgare Miller: - (XIX). AH cult.

Fragaria vesca L.: fresa (XIXbis). AH cult.

Glycyrrhiza glabra L.: orozuz (XVI). AH silv.

Hordeum vulgare L.: alcacer (XIXbis); cebada (XIXbis); harina de cebada (XIXbis). AA cult.

Humulus lupulus L.: lúpulo (XIXbis). AH cult.

Ilex aquifolium L.: acebo (XIXbis). ¿I? silv.

Ipomoea batatas (L.) Lam.: batata de Málaga (XIXbis). AH cult.

Isatis tinctoria L.: - (XIX). I cult.

Juglans regia L.: nogal (XIV); nuez (XVI, XIXbis). AH cult.

Juniperus oxycedrus L.: aceite de enebro (XIXbis); miera (XIXbis). M silv.

Lactarius deliciosus (L.: Fr.) Gray: hongos mizclos o almizcleños (XVII). $\mathrm{AH}$ silv.

Lactuca sativa L.: lechuga (XIV, XVI); (XIX). AH cult.

Laserpitium siler L.: comino rústico (XVI). AH silv.

Lathyrus sativus L.: - (XIX); almorta (XIXbis); harina de almorta (XIXbis). AH cult.

Laurus nobilis L.: laurel (XIXbis). AH cult.

Lavandula latifolia Medik.: espliego (XVIII); espliego en rama y en simiente (XIXbis); esencia de espliego (XIXbis). I silv.

Lens culinaris Medikus: lenteja (XVI, XIXbis). AH cult.
Lepidium sativum L.: mastuerzo (XIX). AH cult.

Lepista nuda (Bull.: Fr.) Cooke o L. personata (Bull.: Fr.) Cooke: seta de pie azul (XVII). AH silv.

Linum usitatissimum L.: - (XIX); aceite de linaza (XIXbis); harina de linaza (XIXbis). I cult.

Lupinus albus L.: lupino o altramuz (XVI); altramuz (XIXbis). AH cult.

Lycopersicon esculentum L.: tomate (XIX, XIXbis). AH cult.

Malus pumila Miller: mançano (XIV); manzana camuesa (XVI); manzana (XIXbis); sidra (XIXbis). AH cult.

Medicago sativa L.: mielga (XIX); alfalfa (XIXbis). AA cult.

Mentha $\times$ gentilis L.: hierbabuena (XVI). AH cult.

Mentha $\times$ rotundifolia (L.) Hudson: mastranzo (XIX); mata los gusanos (XIX). AH silv.

Mespilus germanica L.: níspero (XVI, XVIII). AH cult.

Metroxylon sagu Rottb.: harina de sagú (XIXbis). AH imp.

Morchella esculenta (L.) Pers.: orejas (XVII); cagarrias (XVII); crespilla (XVII). AH silv.

Morus nigra L.: mora (XVI). AH cult.

Myristica fragrans Houtt.: nuez moscada (XVI); macias nuezes (XVII). AH imp.

Nicotiana sp.: tabaco (XVII). M imp.

Olea europaea L.: azeytuna (XIV); aceituna (XVI); - (XIX); aceite común (XIXbis); aceituna aderezada, de Córdoba, de cuquillo, de Sevilla, estrangera, verde común (XIXbis); érrax (XIXbis); hueso de aceituna (XIXbis). AH e I cult.

Opuntia ficus-indica (L.) Miller: higo chumbo (XIXbis). AH cult. 
Origanum vulgare L.: orégano (XIXbis). AH silv.

Oryza sativa L.: arroz (XVI, XVII, XIXbis); harina de arroz (XIXbis). AH cult.

Panicum miliaceum L.: mijo (XIXbis). AA cult.

Papaver somniferum L.: adormidera (XIXbis). M cult.

Pastinaca sativa L.: - (XIX). AH cult.

Petroselinum crispum (Miller) A. W. Hill: perejil (XVI); - (XIX). AH cult.

Phalaris canariensis L.: alpiste (XVI, XIXbis). AA cult.

Phaseolus multiflorus Lam.: - (XIX). AH cult.

Phaseolus vulgaris L.: - (XIX); aluvia (XIXbis); judía verde (XIXbis). $\mathrm{AH}$ cult.

Phoenix dactylifera L.: dátil (XIXbis); dátil de Valencia y estrangero (XIXbis). AH cult. e imp.

Pimpinella anisum L.: - (XIX); anís (XIXbis). AH cult.

Pinus pinea L.: piñón (XVI); piñón en casco y mondado (XIXbis). AH cult.

Piper nigrum L.: pimienta (XVI, XIXbis). AH cult.

Pistacia vera L.: alfónsigo (XVIII); pistacho estrangero, estrangero mondado y mondado (XIXbis). AH cult. e imp.

Pisum sativum L.: arveja (XIV, XVI);(XIX); guisante estrangero en conserva, seco y verde (XIXbis). AH cult. e imp.

Pleurotus eryngii (DC.: Fr.) Quél.: seta de cardo (XVII). AH silv.

Pleurotus eryngii (DC.: Fr.) Quél. var. ferulae Lanzi: [hongo] que naze al pie de las cañahierbas o férulas (XVII). AH silv.

Portulaca oleracea L.: - (XIX). AH cult.
Prunus armeniaca L.: alvarcoque (XIV); albaricoque (XVI); orejón (XVI). AH cult.

P. avium L.: çereza (XIV); cereza (XVI, XIXbis). AH cult.

P. cerasus L.: guinda (XVI). AH cult.

P. dulcis (Miller) D. A. Webb: almendra (XIV, XVI); almendra amarga (XVIII, XIXbis); - (XIX); aceite de almendra (XIXbis); almendra bañada, en casco, fina y ordinaria (XIXbis). AH e I cult.

P. persica (L.) Batsch: albérchiga (XVI); durazno (XIV, XVI); melocotón (XVI); orejón (XVI). AH cult.

Punica granatum L.: granada (XIV, XIXbis); flor de granado (XIXbis). AH y M cult.

Pyrus communis L.: çermeña (XIV); pera (XIV, XVI, XIXbis); - (XIX). AH cult.

Quercus ilex L.: bellota (XVI, XIXbis). AA y AH; silv.

Raphanus sativus L.: -(XIX). AH cult.

Rhus coriaria L.: - (XIX); zumaque en polvo (XIXbis); zumaque en rama (XIXbis). I cult.

Ribes nigrum L.: grosella (XIXbis). $\mathrm{AH}$ cult.

R. uva-crispa L.: agraz (XIV, XIXbis). AH cult.

Rorippa nasturtium-aquaticum Hayek: berro (XVI). AH silv.

Rosa sp.: rrosa (XIV); rosa seca (XIXbis). I cult.

R. gallica L.: rosa castellana (XIXbis). I cult.

Rubia tinctorum L.: - (XIX); rubia molida en la aduana y en los fielatos (XIXbis). I silv.

Rubus ulmifolius Schott: zarzamora (XVI). AH silv.

Rumex acetosa L.: azedera hortense $\mathrm{y}$ silvestre (XVII). AH cult. y silv. 
Saccharum officinarum L.: azúcar (XVI, XIXbis); azúcar común, refinada y en piedra (XIXbis); melaza (XIXbis); melaza de azúcar (XIXbis); rom (XIXbis). AH cult.

Scolymus hispanicus L.: cardillo (XVI). AH silv.

Scorzonera hispanica L.: escorzonera (XIXbis). M silv.

Secale cereale L.: - (XIX); centeno (XIXbis); harina de centeno (XIXbis). AA cult.

Sinapis alba L.: - (XIX); mostaza compuesta (XIXbis). AH cult.

Smilax aspera L.: zarzaparrilla (XVI). M silv.

Solanum dulcamara L.: dulcamara flámula, dulciamara, flámula o sigillo de Santa María (XVII). M silv.

S. melongena L.: - (XIX). AH

S. nigrum L. o $S$. dulcamara L.: yerva mora (XVII). M silv.

S. tuberosum L.: papas o papas del Perú (XVII); - (XIX); patata (XIXbis); sémola de patata (XIXbis). AH cult.

Spinacia oleracea L.: espinaca (XVI). AH cult.

Syzygium aromaticum (L.) Merr. \& Perry: clavo (XVI, XVII); clavillo de especia (XIXbis). AH imp.

Terfezia arenaria (Moris) Trappe: turma de tierra (XVI, XVII); criadilla de tierra (XIXbis). AH silv.

Theobroma cacao L.: cacao (XVII, XIXbis); chocolate (XVII, XIXbis). AH imp.

Thymus vulgaris L. o T. zygis Loefl. ex L.: tomillo (XIXbis). AH silv.

Tribulus terrestris L.: abrojo o tribulo (XVII). AH silv.

Trigonella foenum-graecum L.: alholba (XIXbis). AA cult.
Triticum sp.: trigo trechel (XVII); trigo (XIXbis); harina de bizcocho y de trigo (XIXbis). AH cult.

Triticum aestivum L.: almidón (XVII); trigo de leche o candial (XVII); (XIX). AH e I cult.

Tuber sp.: trufa o criadilla estrangera (XIXbis). AH imp.

Umbilicus rupestris (Salisb.) Dandy: orejas de monje y vasillos, escudetes o escudillos (XVII). M silv.

Veratrum album L.: eleboro blanco (XVII). M silv.

Valeriana officinalis L.: valeriana (XIXbis). AH cult.

Vicia articulata Hornem.: algarroba (XVI, XIXbis); harina de algarroba (XIXbis). AA cult.

V. ervilia (L.) Willd.: yero (XIXbis). AA cult.

V. faba L.: hava (XIV); haba (XVI); (XIX); haba seca y verde (XIXbis). AA y AH cult.

V. sativa L.: arvejón (XVI); - (XIX). AA cult.

Vitis vinifera L.: huva (XIV); pánpano [sarmiento tierno de la vid] (XIV); viña (XIV); pasa (XVI); - (XIX); albillo (XIXbis); chacolí (XIXbis); malvasía (XIXbis); moscatel (XIXbis); mostillo (XIXbis); pasa (XIXbis); pasa de legia, de Málaga, sol, moscatel y estrangera (XIXbis); tinto (XIXbis); uva de Jaén (XIXbis); vinagre (XIXbis); vino (XIXbis); vino común, generoso y estrangero (XIXbis). AH cult. e imp.

Zea mays L.: - (XIX); harina de maiz (XIXbis). AA cult.

Zingiber officinale Roscoe: jenjibre (XVI); gengibre (XVII). AH imp.

Ziziphus jujuba Miller: azufaifa (XVI); (XIX); azofaifa (XIXbis). AH cult. 


\section{Apéndice 2}

\section{Listado de nombres vulgares}

[hongo] que naze al pie de las cañahierbas o férulas (Pleurotus eryngii [DC.: Fr.] Quél. var. ferulae Lanzi) abrojo o tribulo (Tribulus terrestris L.) açafrán (Crocus sativus L.) açanoria (Daucus carota L.) acebo (Ilex aquifolium $\mathrm{L}$.) aceite común (Olea europaea L.) aceite de almendra (Prunus dulcis [Miller] D. A. Webb) aceite de coco (Cocos nucifera L.) aceite de enebro (Juniperus oxycedrus L.) aceite de linaza (Linum usitatissimum L.) aceituna, aceituna aderezada, de Córdoba, de cuquillo, de Sevilla, estrangera, verde común (Olea europea L.) acelga (Beta vulgaris L.) acerola (Crataegus azarolus L.) achicoria (Cichorium intybus L.) achote (Bixa orellana L.) adormidera (Papaver somniferum L.) agraz (Ribes uva-crispa L.) ajo, ajo seco (Allium sativum L.) alazor (Carthamus tinctorius L.) albaricoque (Prunus armeniaca L.) albérchiga (P. persica [L.] Batsch) albillo (Vitis vinifera $\mathrm{L}$.) alcacer (Hordeum vulgare L.) alcachofa (Cynara scolymus L.) alcaparra (Capparis spinosa L.) alcarabea (Carum carvi L.) alfalfa (Medicago sativa L.) alfónsigo (Pistacia vera $\mathrm{L}$.) algarroba (Vicia articulata Hornem.) alholba (Trigonella foenum-graecum L.) almendra, almendra amarga, almendra bañada, en casco, fina y ordinaria (Prunus dulcis [Miller] D. A. Webb) almidón (Triticum aestivum L.) almorta (Lathyrus sativus L.) alpiste (Phalaris canariensis L.) altramuz (Lupinus albus L.) aluvia (Phaseolus vulgaris L.) alvarcoque (Prunus armeniaca L.) anís (Pimpinella anisum L.) árnica (Arnica montana L.) arroz (Oryza sativa L.) arveja (Pisum sativum L.) arvejón (Vicia sativa L.) avellana, avellana en casco, avellana tostada y mondada (Corylus avellana L.)

avellano (C. avellana $\mathrm{L}$.) avena (Avena sativa $\mathrm{L}$.) azafrán (Crocus sativus L.) azedera hortense y silvestre (Rumex acetosa $\mathrm{L}$.)

azerola (Crataegus azarolus L.)

azeytuna (Olea europaea L.) azofaifa (Ziziphus jujuba Miller) azúcar, azúcar común, refinada y en piedra (Saccharum officinarum L.)

azufaifa (Ziziphus jujuba Miller) batata de Málaga (Ipomoea batatas [L.] Lam.)

bellota (Quercus ilex L.)

berro (Rorippa nasturtium-aquaticum [L.] Hayek) berza (Brassica oleracea L.) 
besicos de monja (Cardiospermum halicacabum L.)

breva (Ficus carica L.)

cacahué, cacahuét (Arachis hypogaea L.)

cacao (Theobroma cacao L.)

café (Coffea arabica L.)

cagarrias (Morchella esculenta [L.] Pers.)

calabaza (Cucurbita maxima Duchesne ex Lam.)

calabazate (C. maxima Duchesne ex Lam.)

calisaya (Cinchona calisaya Wedd.)

canela, canela de Ceilán, de Holanda, de

la China y de Manila (Cinnamomum verum J. Presl)

canela de Icabo (Canella winterana [L.]

Gaertner)

cañamón (Cannabis sativa L.)

cardillo (Scolymus hispanicus L.)

cardo (Cynara cardunculus L.)

cardón (Agave americana L.)

carne de membrillo (Cydonia oblonga Miller)

castaña, castaña mondada y apilada, castaña verde (Castanea sativa L.)

cebada (Hordeum vulgare L.)

cebolla (Allium cepa L.)

centeno (Secale cereale L.)

cereza, çereza (Prunus avium L.)

çermeña (Pyrus communis L.)

chacolí (Vitis vinifera L.)

chicoria amarga (Cichorium intybus L.)

chirimoya (Annona cherimola Miller)

chocolate (Theobroma cacao L.)

chufa (Cyperus esculentus L.)

cidra (Citrus medica L.)

cilantro (Coriandrum sativum L.)

clavillo de especia (Syzygium aromaticum

[L.] Merr. \& Perry)

clavo (S. aromaticum [L.] Merr. \& Perry)

coca de Levante para matar peces (Anamirta cocculus [L.] Wight \& Arn.)

cogombro, cohombro (Cucumis melo L. subsp. flexuosus [L.] Grab.) col (Brassica oleracea L.)

coliflor (B. oleracea L.)

comino (Cuminum cyminum L.)

comino rústico (Laserpitium siler L.)

crespilla (Morchella esculenta [L.] Pers.)

criadilla de tierra (Terfezia arenaria [Mo-

ris] Trappe)

culantrillo (Adiantum capillus-veneris L.)

culantro (Coriandrum sativum L.)

dátil (Phoenix dactylifera $\mathrm{L}$.)

dátil de Valencia y estrangero ( $P$. dactylifera $\mathrm{L}$.)

diacitrón (Citrus medica L.)

dulcamara flámula, dulciamara, flámula o sigillo de Santa María (Solanum dulcamara L.)

durazno (Prunus persica [L.] Batsch)

eleboro blanco (Veratrum album L.)

érrax (Olea europaea L.)

escarola (Cichorium endivia L.)

escorzonera (Scorzonera hispanica L.)

esencia de espliego (Lavandula latifolia Medik.)

espárrago, espárragos de Calera, de jardín, de la Mancha y de Seseña (Asparagus officinalis $\mathrm{L}$.)

espárrago de Trujillo (A. acutifolius L.)

espinaca (Spinacia oleracea L.)

espliego, espliego en rama y en simiente

(Lavandula latifolia Medik.)

figo (Ficus carica L.)

flor de alazor (Carthamus tinctorius L.)

flor de cardo (Cynara cardunculus L.)

flor de granado (Punica granatum L.)

fresa (Fragaria vesca L.)

fruta en piña de América (Ananas comosus [L.] Merr.)

garbanzo, garbanzo tostado (Cicer arietinum L.)

garrofa (Ceratonia siliqua L.)

garvanço (Cicer arietinum L.)

gengibre (Zingiber officinale Roscoe)

granada (Punica granatum L.)

grosella (Ribes nigrum L.) 
guinda (Prunus cerasus L.)

guisante estrangero en conserva, seco y verde (Pisum sativum L.)

haba, haba seca y verde (Vicia faba L.)

harina de algarroba ( $V$. articulata Hornem.)

harina de almorta (Lathyrus sativus L.)

harina de arroz (Oryza sativa L.)

harina de bizcocho y de trigo (Triticum $\mathrm{sp}$.)

harina de cebada (Hordeum vulgare L.)

harina de centeno (Secale cereale L.)

harina de linaza (Linum usitatissimum L.)

harina de maiz (Zea mays L.)

harina de sagú (Metroxylon sagu Rottb.)

hava (Vicia faba L.)

hierba para cuajar (Cynara cardunculus L.)

hierbabuena (Mentha $\times$ gentilis L.)

higo, higo estrangero, higo verde, higos pasos de la tierra, de Málaga, de Murcia y de Valencia (Ficus carica L.)

higo chumbo (Opuntia ficus-indica [L.] Miller)

hoja de naranjo (Citrus $\times$ aurantium L.)

hoja de sen (Cassia obovata Collad.)

hongo de olmos (Agrocybe aegerita [Brig.] Singer o Pleurotus ostreatus [Jacquin: Fr.] Kummer

hongos mizclos o almizcleños (Lactarius deliciosus [L.: Fr.] Gray)

hueso de aceituna (Olea europaea L.)

huva (Vitis vinifera $\mathrm{L}$.)

jenjibre (Zingiber officinale Roscoe)

judía verde (Phaseolus vulgaris L.)

laurel (Laurus nobilis L.)

lechuga (Lactuca sativa L.)

lenteja (Lens culinaris Medikus)

lima, limas de Murcia y de la Vera ( $\mathrm{Ci}$ trus aurantifolia [Christm.] Swingle)

limón $(C . \times$ limon [L.] Osb. $)$

limones de Murcia y de la Vera $(C . \times$ limon [L.] Osb.)

lobitos (Boletus sp.) lupino o altramuz (Lupinus albus L.)

lúpulo (Humulus lupulus L.)

macias nuezes (Myristica fragrans

Houtt.)

madroño (Arbutus unedo L.)

malvasía (Vitis vinifera $\mathrm{L}$.)

mançano (Malus pumila Miller)

manzana, manzana camuesa (M. pumila Miller)

mastranzo (Mentha $\times$ rotundifolia [L.] Hudson)

mastuerzo (Lepidium sativum L.)

mata los gusanos (Mentha $\times$ rotundifolia

[L.] Hudson)

melaza, melaza de azúcar (Saccharum officinarum $\mathrm{L}$.)

melocotón (Prunus persica [L.] Batsch)

melón, melones de Añover, de la tierra de

Madrid, de Madrid y de Valencia

(Cucumis melo L.)

membrillo (Cydonia oblonga Miller)

mielga (Medicago sativa L.)

miera (Juniperus oxycedrus L.)

mijo (Panicum miliaceum L.)

mora (Morus nigra L.)

moscatel (Vitis vinifera $\mathrm{L}$.)

mostaza compuesta (Sinapis alba L.)

mostillo (Vitis vinifera $\mathrm{L}$.)

nabo (Brassica napus L.)

naranja (Citrus $\times$ aurantium L.)

naranjas de Murcia y de la Vera $(C . \times$ aurantium L.)

níspero (Mespilus germanica L.)

nogal (Juglans regia L.)

nuez ( $J . r$. L.)

nuez moscada (Myristica fragrans Houtt.)

orégano (Origanum vulgare L.)

orejas (Morchella esculenta [L.] Pers.)

orejas de monje y vasillos, escudetes o escudillos (Umbilicus rupestris [Salisb.] Dandy)

orejón (Prunus armeniaca L.)

orejón (P. persica [L.] Batsch)

orozuz (Glycyrrhiza glabra L.) 
palmito (Chamaerops humilis L.)

palo de las Indias (Caesalpinia paraguariensis [Parodi] Burkart)

pánpano [sarmiento tierno de la vid] (Vitis vinifera $\mathrm{L}$.)

papas o papas del Perú (Solanum tuberosum $\mathrm{L}$.)

pasa (Vitis vinifera $\mathrm{L}$.)

pasa de legia, de Málaga, sol, moscatel y estrangera $(V . v$. L. $)$

patata (Solanum tuberosum L.)

pepino, pepino estrangero en vinagre (Cucumis sativus L.)

pera (Pyrus communis L.)

perejil (Petroselinum crispum [Miller] A. W. Hill)

pimienta (Piper nigrum L.)

pimiento, pimiento de Indias, pimiento de la Rioja, pimiento molido (Capsicum annuum L.)

piñón (Pinus pinea L.)

piñón en casco y mondado ( $P . p$. L.)

pistacho estrangero, estrangero mondado y mondado (Pistacia vera $\mathrm{L}$.)

pita zarza (Agave americana L.)

puerro (Allium porrum L.)

rábano (Brassica rapa $\mathrm{L}$.)

repollo (B. oleracea L.)

rom (Saccharum officinarum L.)

rosa castellana (Rosa gallica L.)

rosa seca (Rosa sp.)

rrosa $(R . \mathrm{sp}$.)

rubia molida en la aduana y en los fiela-

tos (Rubia tinctorum L.)

sémola de patata (Solanum tuberosum L.)

seta de cardo (Pleurotus eryngii [DC.:

Fr.] Quél.) seta de pie azul (Lepista nuda [Bull.: Fr.]

Cooke o L. personata [Bull.: Fr.] Cooke) setas estrangeras (Boletus sp.)

sidra (Malus pumila Miller)

tabaco (Nicotiana sp.)

taragontía (Dracunculus vulgaris Schott) té (Camellia sinensis [L.] Kuntze)

tinto (Vitis vinifera $\mathrm{L}$.)

tomate (Lycopersicon esculentum L.)

tomillo (Thymus vulgaris L. o T. zygis

Loefl. ex L.)

toronja (Citrus maxima [Burm.] Merrill)

tragontía o serpentaria mayor (Dracuncu-

lus vulgaris Schott)

trigo, trigo de leche o candial (Triticum

sp.)

trigo trechel (Triticum sp.)

trufa o criadilla estrangera (Tuber sp.)

turma de tierra (Terfezia arenaria [Moris]

Trappe)

uva de Jaén (Vitis vinifera L.)

valeriana (Valeriana officinalis L.)

vinagre (Vitis vinifera $\mathrm{L}$.)

vino $(V . v . \mathrm{L}$.

vino común, generoso y estrangero $(V . v$.

L.)

viña $(V . v$. L.)

yero (Vicia ervilia [L.] Willd.)

yerva mora (Solanum nigrum L. o $S$. dulcamara L.)

zafranromín almocaser (Carthamus tinctorius L.)

zanahoria (Daucus carota L.)

zarzamora (Rubus ulmifolius Schott)

zarzaparrilla (Smilax aspera L.)

zumaque en polvo, zumaque en rama

(Rhus coriaria $\mathrm{L}$.) 